\title{
Review
}

\section{Retinoid-induced apoptosis in normal and neoplastic tissues}

\author{
Laszlo Nagy ${ }^{1,3,4}$, Vilmos A. Thomazy ${ }^{1}$, Richard A. Heyman ${ }^{2}$ \\ and Peter J.A. Davies ${ }^{1,3}$ \\ ${ }^{1}$ Department of Pharmacology, University of Texas-Houston, Medical School, \\ Houston, Texas 77225 USA \\ 2 Ligand Pharmaceuticals, San Diego, California, 92121 USA \\ ${ }^{3}$ Corresponding author: PJAD, tel: 713-500-7480; fax: 713-500-7455; \\ e-mail: pdavies@farmr1.med.uth.tmc.edu \\ 4 Present address for correspondence: The Salk Institute for Biological Studies, \\ Gene Expression Laboratory, La Jolla, California 92037; \\ tel: (619) 453-4100 fax:(619) 455-1349; e-mail: Inagy@axp1.salk.edu
}

Received 18.8.97; revised 19.9.97; accepted 22.9.97

Edited by M. Piacentini

\begin{abstract}
Vitamin $A$ and its derivatives (collectively referred to as retinoids) are required for many fundamental life processes, including vision, reproduction, metabolism, cellular differentiation, hematopoesis, bone development, and pattern formation during embryogenesis. There is also considerable evidence to suggest that natural and synthetic retinoids have therapeutical effects due to their antiproliferative and apoptosis-inducing effects in human diseases such as cancer. Therefore it is not surprising that a significant amount of research was dedicated to probe the molecular and cellular mechanisms of retinoid action during the past decade. One of the cellular mechanisms retinoids have been implicated in is the initiation and modulation of apoptosis in normal development and disease. This review provides a brief overview of the molecular basis of retinoid signaling, and focuses on the retinoid-regulation of apoptotic cell death and gene expression during normal development and in pathological conditions in vivo and in various tumor cell lines in vitro.
\end{abstract}

Keywords: retinoic acid; retinoic acid receptor; RAR; RXR; apoptosis; tissue transglutaminase; gene expression

Abbreviations: ATRA, All-trans retinoic acid; 9-cis RA, 9-cis retinoic acid; $P G-J_{2}, 15-$ deoxy- $\Delta{ }^{12,14} P \mathrm{PJ}_{2}$; RAR, retinoic acid receptor; $\mathrm{RXR}$, retinoid $X$ receptor; Tgase, tissue transglutaminase; SMRT, silencing mediator of retinoid and thyroid receptors; N-CoR, nuclear receptor corepressor; CREB, creb binding protein; HDAC-1, histone deacetylase-1

\section{Retinoids are modulators of transcription}

The retinoid's mechanism of action in both normal and pathological conditions has been the subject of more than a decade of intense research. The cloning and discovery of the retinoic acid receptor (RAR), which belongs to the superfamily of ligand-activated transcription factors (nuclear receptors) revolutionized our understanding as to how retinoids exert their pleiotropic effects (for reviews see Chambon (1996); Mangelsdorf et al (1994)). Members of the nuclear receptor superfamily mediate the biological effects of many hormones, vitamins and drugs (i.e. steroid hormones, thyroid hormones, vitamin $\mathrm{D}$, prostaglandin- $\mathrm{J}_{2}\left(P G-\mathrm{J}_{2}\right)$ and drugs that activate peroxisomal proliferation). There are two families of retinoid receptors, Retinoid X Receptors (RXRs) that bind 9-cis retinoic acid (9-cis RA) and Retinoic Acid Receptors (RARs) that bind both 9-cis RA and all-trans retinoic acid (ATRA) (for reviews see Chambon 1996; Mangelsdorf et al, 1994)). Each of these receptor families includes at least three distinct genes, (RAR $\alpha, \beta$ and $\gamma ; \operatorname{RXR} \alpha, \beta$ and $\gamma)$ that through differential promoter usage and alternative splicing, give rise to a large number of distinct retinoid receptor proteins (for reviews see Chambon 1996; Mangelsdorf et al, 1994).

There is a clear functional distinction between the RXRs and the RARs. Nuclear receptors generally function as dimers; steroid receptors form homodimers while most of the other members of the superfamily form heterodimers with other nuclear receptors. RXRs form heterodimers with several nuclear receptors including thyroid hormone receptors (TR), the vitamin $D$ receptor (VDR) and the receptors for the peroxisomal proliferator drugs and $P G-J_{2}$ (PPARs). RXRs also heterodimerize with the RARs and can homodimerize as well (Chambon, 1996; Mangelsdorf et al, 1994). Because of the multiplicity of RXR partners, ligands that activate RXRs can have biological activity via diverse endocrine signaling pathways. The function of RARs is more restricted. RARs do not form homodimers but they do bind to RXRs. Generally ligand activation of both the RAR and RXR component of RAR/RXR heterodimers can contribute to the activity of the receptors (Roy et al, 1995) although there are some situations where ligand activation of either component alone is sufficient for a full biological response (Kurokawa et al, 1994; Nagy et al, 1995).

The role of ligands in the regulation of retinoid receptor function is complex. Unliganded receptors bind to the retinoid-response elements of retinoid-regulated genes, and recruit transcription factors that negatively regulate gene expression (Chen and Evans, 1995; Horlein et al, 1995). Thus unliganded receptors can act as negative transcription factors. Retinoids bind to a ligand-binding site in the carboxyl-terminal half of the RARs (E/F domain) causing marked conformational changes (Bourguet et al, 1995; Renaud et al, 1995) by releasing the negative regulatory factors (corepressors) and/or by facilitating the recruitment of positive regulators (coactivators) of gene expression (Kurokawa et al, 1995) (Figure 1). This way ligands can directly activate the expression of target genes by either relieving negative control or facilitating the activity of 
positive transcription factors. In addition to their direct effects on transcription, ligand-activated RARs modulate the activity of other transcription factors such as AP-1 (this is termed cross-coupling) (Schule et al, 1991). Activated RARs inhibit the activity of the transcription factors and thereby control the expression of AP-1 regulated genes. Thus there are two ways that retinoids can regulate gene expression, either by direct effects on transcription or in an indirect effect on AP-1 activity. The first mechanism will be dependent on RRE' $s$ within the target genes whereas the latter mechanism will not. A surprising development in retinoid biology has been the recent discovery that the two mechanisms for the control of gene expression by retinoids are the basis of different biological responses. The antiproliferative effects of retinoids are linked to the inhibition of AP-1 activity (Chen et al, 1995; Fanjul et al, 1994; Nagpal et al, 1995) whereas the induction of cellular differentiation depends on the direct activation of transcription of specific retinoid-regulated genes. Synthetic retinoids, such as SR11220 (Fanjul et al, 1994), have been developed that selectively activate the AP-1 inhibitory domains of retinoid receptors without activating transcription. These compounds provide powerful pharmacologic tools for identifying AP-1 dependent aspects of retinoid activity in vivo.

The recent discovery of nuclear receptor associated proteins (cofactors $=$ coactivators and corepressors) provides us with detailes as to how DNA bound unliganded and liganded receptor dimers influence transcription of target genes both by direct and indirect mechanisms. For the direct transcriptional effects a simple binary paradigm is emerging from these studies (Figure 1). Unliganded receptors bind to response elements of target genes and repress transcription through recruitment of a repressor complex containing corepressors (SMRT/N-CoR), Sin3 and histone deacetylases (HDAC-1 and 2) (Alland et al, 1997; Heinzel et al, 1997; Nagy et al, 1997b). This probably leads to histone deacetylation and formation of an inactive chromatin structure preventing transcription. Ligand binding causes the dissociation of corepressor proteins and promotes association of coactivators with liganded receptors (Figure 1). Interestingly, several of the so far identified coactivator proteins have histone acetylase activity i.e. CBP/p300 (Ogryzko et al, 1996) and ACTR (Chen et al, 1997) which contributes to the formation of an active chromatin structure and results in the transcription of the target gene. Remarkably, several of the coactivators and corepressors are shared by multiple signaling pathways (i.e. CBP has been implicated in AP-1, pS3, STAT signaling among others and Sin3, HDAC-1 are involved in Mad-Max signaling (Ayer et al, 1995; Hassig et al, 1997; Kamei et al, 1996). This raises the possibility that formation of regulatory (activator or repressor) complexes and/or competition for limiting amounts of these proteins may prove to be critical in determining which signaling pathway can be activated in a given cell at a given time. This model of transcriptional repression and activation by nuclear receptors and their cofactors provides a direct link not only between multiple signaling pathways critical in cellular proliferation, differentiation and apoptosis but also between these pathways and the chromatin structure of target genes. It is likely though that there are additional mechanisms (i.e. phosphorylation, direct interactions between receptors/cofactors and the basal transcriptional machinery) associated with cofactor/receptor function which may contribute to the fine tuning of transcriptional regulation by nuclear receptors.

\section{Role of retinoid receptors in vivo}

Despite the strong evidence for the specialized functions of RARs drawn from transfection and cultured cell studies, experiments carried out in whole animals have suggested that the relationship between the functions of the individual RARs may be much more complex (for a review see Kastner et al (1995)). Deletion of individual RARs in mice by homologous recombination (receptor knock-outs) have produced surprisingly modest phenotypic consequences. The RAR $\beta$-null genotype is without an apparent phenotype (Luo et al, 1995). The knock-out of RAR $\alpha 1$, the predominant RAR $\alpha$ isoform in the embryo, has no discernable phenotype (Li et al, 1993; Lufkin et al, 1993). Deletion of both RAR $\alpha$ isoforms, the RAR $\alpha$-null genotype, showed normal embryonic viability but early post-natal death. The RAR $\alpha$-null animals are sterile due

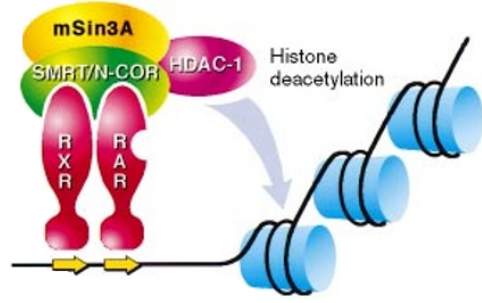

REPRESSED

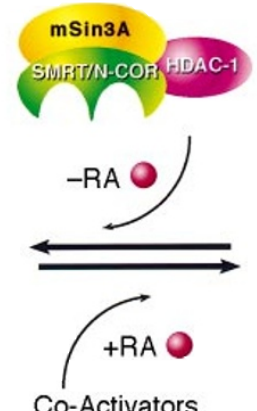

Co-Activators

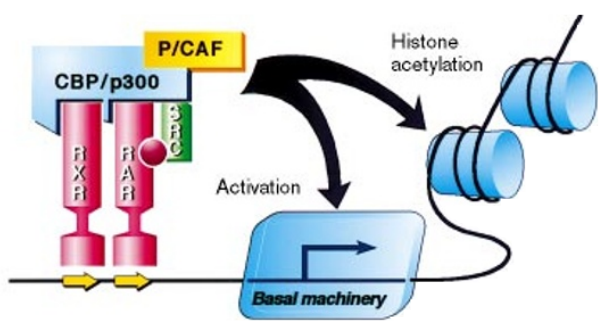

ACTIVE

Figure 1 Hormonal targeting of nuclear complexes to chromatin template. In the absence of hormone, a SMRT, Sin3A and HDAC1 complex associates with unliganded receptor heterodimers. In this complex histone deacetylase activity creates a repressive chromatin environment. Addition of hormone triggers the release of the repressor complex and recruitment of co-activators that include histone acetylases such as CBP/p300 and P/CAF. This results in local acetylation of histones the recruitment of the basal transcription machinery and transcriptional activation. (Adapted from Perlmann and Evans, (1997)) 
to testis degeneration and they also showed partial syndactyly (evidence for deficient cell death in the interdigital webs of the developing limbs) (Lufkin et al, 1993). Deletion of the RAR $\gamma 2$ isotype is without evident phenotype whereas knock-out of both $\mathrm{RAR} \gamma$ isoforms, the RAR $\gamma$-null phenotype, has normal embryonic viability but early post-natal mortality due to growth retardation. These animals are sterile due to prostatic abnormalities, show subtle changes in vertebral patterning and also show partial syndactyly (Kastner et al, 1995; Lohnes et al, 1993). In the context of intact physiological systems, deficits in the function of individual RARs appear to be compensated for by the activity of the residual RARs present in the affected tissues. This is likely to be the case since double knock-outs, particularly of RARs $\alpha$ and $\gamma$, produce a much more severe phenotype than the single receptor knockouts (Lohnes, 1994; Mendelsohn, 1994). The phenotype of the RAR $\alpha, \gamma$-double null animals replicates most of the teratogenic effects of vitamin A deficiency (VAD) (Wilson et al, 1953; Wolbach and Howe, 1925) suggesting that the combined activity of these two receptors is central to the morphogenic effects of retinoids.

The results obtained with retinoid receptor knock-out animals suggest that there may be redundancies in the function of RARs in vivo. This redundancy may reflect the fact that in vivo, different receptors may contribute to the regulation of the same genes, i.e. the level of transcription of those genes reflects the total level of receptors and ligands present in the tissue rather than the presence of any particular receptor (Kastner et al, 1995). This model of redundancy is supported by the observation that defects in retinoid-regulated gene expression in F-9 cells rendered null for RAR $\alpha$ can be partially reversed by over-expression of RAR $\gamma$ (Taneja et al, 1995). Similar results have been obtained in studies of myeloid (HL-60) differentiation (Robertson et al, 1992a,b). A retinoid-resistant cell line (HL-60R) was developed by Collins and associates. This cell line has a mutant allele of $\operatorname{RAR} \alpha(\operatorname{RAR} \alpha 411)$ which is a truncated, dominant negative transcription factor inhibiting retinoid signaling. Overexpression of either RARs or RXRs restores retinoid responsiveness in these cells (Robertson et al, 1992b).

An alternative explanation for the apparent redundancy in RAR function in vivo is that it may represent overlapping biological responses. Each RAR may preferentially regulate specific retinoid-regulated genes that co-operate in producing a full biological response. The function of the individual receptor may be sufficient to partially complete the process but it is not as effective as the activity of both receptors working in tandem. In the context of apoptosis occurring during limb development, it is possible that the effects of $\operatorname{RAR} \beta$ may be directly on the apoptotic cells in the interdigital webs (where it is expressed =cell autonomous) whereas the effects of RAR $\gamma$, which is expressed in adjacent connective tissues, may involve the induction of cytokines that trigger the death of the cells in the interdigital webs (non cell-autonomous). Under normal circumstances both mechanisms may contribute to the death of the interdigital tissues but in the experimental situation deletion of one of the pathways results in a partial block in apoptosis. This model is supported by the observation that both the RAR $\alpha$ - and RAR $\gamma$-null phenotypes include partial syndactyly (Kastner et al, 1992).

\section{Retinoid receptors and apoptosis}

Retinoids have long been recognized to have major effects on cellular proliferation and differentiation. Retinoids have a broad spectrum of anti-proliferative activity in cultured cell systems, particularly transformed cells and are used in the therapy of certain hyper-proliferative, pre-malignant and malignant diseases (Gudas et al, 1994). In addition retinoids alter the differentiation of many cell types, inhibiting squamous differentiation in epithelial cells (Jetten et al, 1990) and inducing the differentiation of myeloid cells (Robertson et al, 1992b). These activities underlie their use in the treatment of leukemias and certain squamous cancers. Recently it has become recognized that retinoids also regulate the expression of programmed cell death, inducing the death of certain cell types (Martin et al, 1990) and inhibiting apoptosis in others (Yang et al, 1993).

The link between retinoids and cell death was an outgrowth of studies on the cellular basis of retinoid teratogenesis. Retinoids are potent teratogens producing a complex array of malformations in skeletal and neural crest-derived structures (Kochar, 1967). In a series of studies carried out by Sulik and co-workers it was demonstrated that one of the reasons for retinoid-induced teratogenesis was the expansion of zones of physiological cell death (Sulik et al, 1988). This was particularly true in the limb, where expanded zones of apoptosis in the interdigital tissues and excessive death in the patternforming ectoderm (apical ectodermal ridge) lead to complex limb malformations (Alles and Sulik, 1989; Sulik and Dehart, 1988). Similar effects of retinoids have been reported in !ower vertebrates (Ferretti and Geraudie, 1995). Administration of retinoids to limb apoptosisdefective mice (hammertoe strain) lead to partial restoration of interdigital apoptosis (Zakeri and Ahuja, 1994) and exogenous retinoids applied to limb explants or cultured interdigital cells cause extensive apoptosis (Jiang and Kochhar, 1992; Lee et al, 1994). Similar mechanisms contribute to the cranio-facial teratogenesis of retinoids (Sulik et al, 1988) and their effects on development of the CNS (Alles and Sulik, 1990; Sulik et al, 1988).

In addition to their effects on embryonic tissues retinoids have also been implicated in the induction of cell death in many tumor-derived cultured cell systems. Retinoids-induce apoptosis in myeloid leukemia cells (Martin et al, 1990), neuroblastoma cells (Melino et al, 1994), breast (Seewald et al, 1995), ovarian (Krupitza et al, 1995) and cervical cancer cells (Oridate et al, 1995) and many other types of cells (Atencia et al, 1994; Corbeil et al, 1994; Kalemkerian et al, 1995; Nakamura et al, 1995). In many of these systems ligands that activate particular subsets of RARs have proven to be particularly efficacious. Apoptosis of tracheal epithelial cells is preferentially induced by RAR $\alpha$ agonists (Zhang et al, 1995), apoptosis of thymic lymphocytes responds best to RAR $\gamma$ ligands (Szondy et al, 1997), co-stimulation of RAR $\alpha$ and RAR $\gamma$ induces apoptosis in neuroblastoma cells (Melino et al, 1997), and 
ligand activation of $\operatorname{RAR} \beta$ may play a particularly important role in limb teratogenesis (Soprano et al, 1994). We have recently shown that $R X R$ ligands can also be very effective inducers of apoptosis in myeloid leukemia cells (Nagy et al, 1996b; Nagy et al, 1995). Our understanding of retinoidinduced apoptosis in tumor cells has been complicated by the recent observation that some of the most active inducers of tumor cell apoptosis, such as 4-hydroxyphenylretinamide are not active in RAR-signaling pathways suggesting that alternative pathways of retinoid signaling may be critical for some forms of apoptosis (Sheikh et al, 1995). This issue is still unresolved though because there are other reports claiming that this compound acts through RAR $\gamma$ (Fanjul et al, 1996).

At present it is not known to what extent these observations represent the heterogeneity of factors that can induce apoptosis in cultured cells, particularly tumor cells, as opposed to reflecting the true complexity of retinoid-regulated apoptosis as it occurs in vivo.

While there is substantial evidence that extrinsic retinoids administered to animals can be highly teratogenic due at least in part to the induction of cell death, it is much less clear whether the retinoids normally present in tissues, endogenous retinoids, play a physiological role in regulating cell death. The question can be best addressed by administering retinoid antagonists to animals and observing effects on normal embryonic development. Unfortunately until recently potent and specific retinoid antagonists have not been available so this experimental approach has not been explored. The recent development of RAR-antagonists, AGN 193109 a very potent pan-RAR antagonist (Johnson et al, 1995) and Ro13-5320, an RAR $\alpha$ selective antagonist (Apfel et al, 1992), have made such experiments feasible.

Although RAR antagonists have not been available, two other approaches, deletion of individual retinoid receptors and the induction of vitamin A deficiency, have provided critical information on the role of retinoids as physiological regulators of apoptosis. The Chambon laboratory has carried out comprehensive studies on morphogenesis and cell death in animals in which individual retinoid receptors have been deleted by homologous recombination (receptor knock-out animals, reviewed in (Kastner et al, 1995). Single receptor knock-outs have a limited phenotype. However partial syndactyly (a failure of regression of interdigital tissues due to defective apoptosis) was a common feature of animals null for either RAR $\alpha$ or RAR $\gamma$ (Kastner et al, 1995) suggesting that either or both receptors were essential for normal cell death in this tissue. These studies demonstrate that retinoid receptors are crucial for normal apoptosis in the limb but do not answer the question of whether the ligands for these receptors, i.e. endogenous retinoids, are also required.

The role of endogenous retinoids in normal morphogenesis has also been approached by making animals retinoid deficient via dietary deprivation (the vitamin A deficiency syndrome - VAD). A series of studies in rodents carried out in the late 1940s established that VAD is very teratogenic, resulting in malformations in the eye, cardiovascular system and uro-genital system. Limb development abnormalities were not noted (Wilson et al, 1953). Unfortunately these studies preceded the recognition of apoptosis as a component of normal morphogenesis and so the findings were discussed in terms of abnormal patterns of cellular differentiation rather than the possible failures in normal tissue regression. Since that time there is no evidence in the literature to suggest that the association between VAD and apoptosis has been systematically addressed.

The available evidence suggests that retinoids are involved in the regulation of apoptosis but they shed little information on how these effects occur.

\section{Retinoid regulated gene expression during apoptosis: regulation of tissue transgluta- minase}

Retinoids produce their biological effects by alterations in patterns of gene expression. Retinoids have been shown to regulate the expression of regulatory factors of apoptosis such as p21 (Lin et al, 1996) (Boccia et al, 1997) and Bcl-2 (Nagy et al, 1996b) as well as effector enzymes such as transglutaminases (Chiocca et al, 1988) and sphingomyelinases (Riboni et al, 1995) which have been implicated in the induction and execution of cell death. The normal and external retinoid- induced in vivo expression pattern of one of the retinoic acid receptors (RAR $\beta$ ) suggested that it may be associated with cell death also (Mendelsohn et al, 1991). Analysis of the expression pattern of RAR $\beta$ in embryos from mothers treated with teratogenic doses of ATRA, indicated that mRAR-beta 2 promoter is selectively induced. These findings suggest that overexpression of the mRAR-beta 2 isoform is involved in RA-generated malformations. This raises the possibility that mRAR-beta 2 may have a role in development of the limbs, as an inhibitor of cartilage formation, in programmed cell death and in the formation of loose connective tissue.

One of the most extensively studied systems is the differentiation and subsequent apoptosis of the myeloid leukemia cell line (HL-60). The initial observation that retinoids induce apoptosis subsequent of cellular differentiation was made by Martin et al (1990). All-trans retinoic acid (ATRA) induces differentiation of HL-60 cells toward mature neutrophil granulocytes which subsequently die by apoptotic cell death (Figure 2). Using receptor selective retinoids we were able to demonstrate that the biological response induced by ATRA a pan-receptor agonist (under tissue culture conditions) has two components. The first one is an RAR $\alpha$-induced differentiation phase which is followed by an RXR-ligand-dependent apoptotic phase. If one assumes that the receptor species mediating the effects of the panagonist and RXR-specific retinoids on apoptosis is also an RAR/RXR heterodimer, then it is likely that ligand activation of the RXR moiety of this complex alters the transciption of genes critical to the induction of apoptosis. Alternatively, ligand-activation of different RXR/ nuclear receptor heterodimers or RXR homodimers may regulate the apoptotic response. There are several changes in the levels of expression of numerous apoptosis related genes during the retinoid-induced death of HL-60 cells. The level of the anti-apoptotic protein, Bcl-2 is down regulated (Nagy et al, 1996b) whilst the expression levels of effector enzymes such as tissue transglutaminase (Nagy et al, 

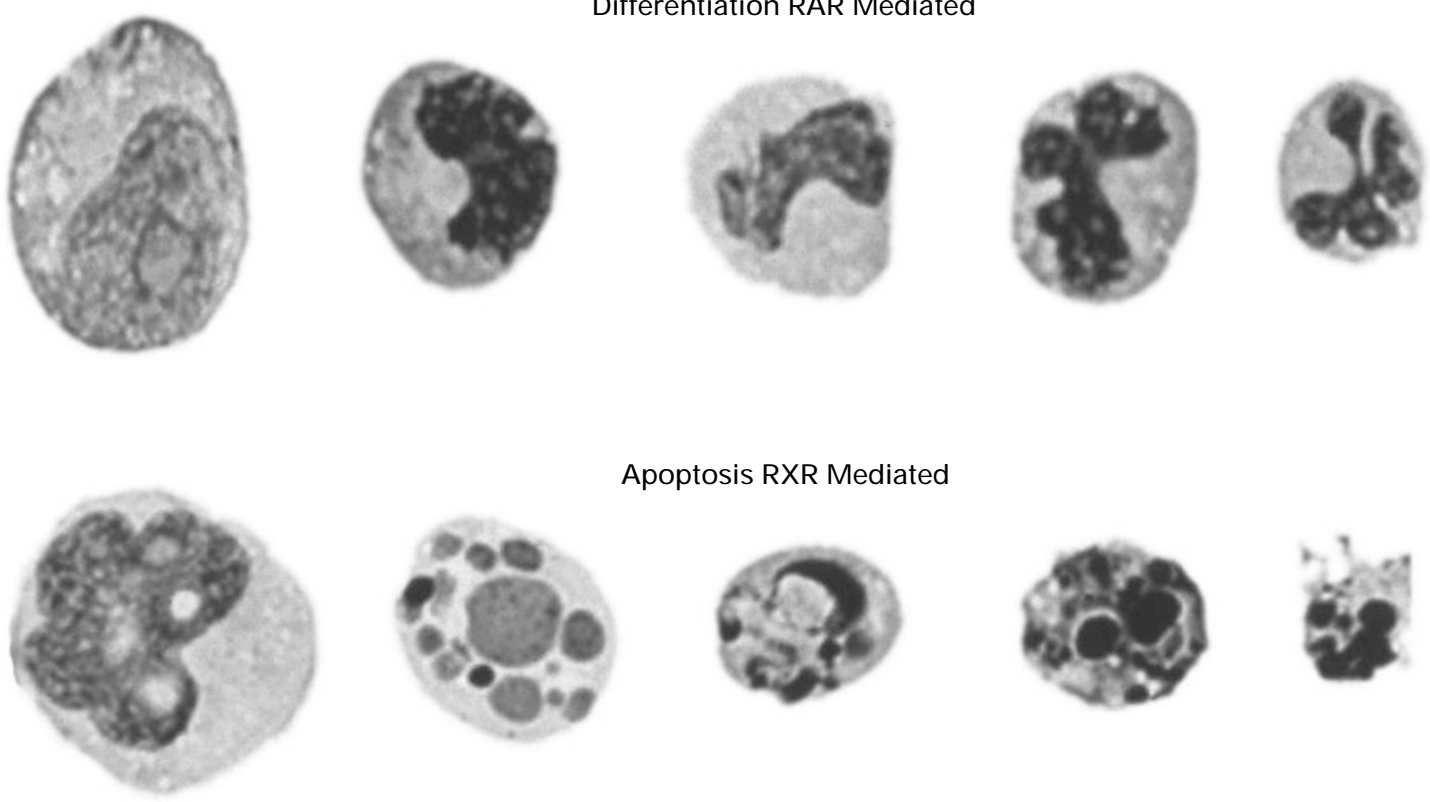

Apoptosis RXR Mediated

Figure 2 Retinoid regulation of differentiation and apoptosis in myeloid leukemia cells (HL-60). HL-60 cell undergo retinoic acid induced differentiation and subsequently die by apoptotic cell death. Upper panel: typical morphology of cells undergoing granulocytic differentiation (an RAR mediated process): myeloblast, metamyelocyte, band, and segmented polymorphonuclear cells (from left to right). Lower panel: morphological changes associated of HL-60 cells undergoing apoptosis (an RXR mediated effect): differentiated cell, nuclear fragmentation, and nuclear and cytoplasmic fragmentation and condensation, apoptotic remnant (from left to right)

A

Activation of RAR

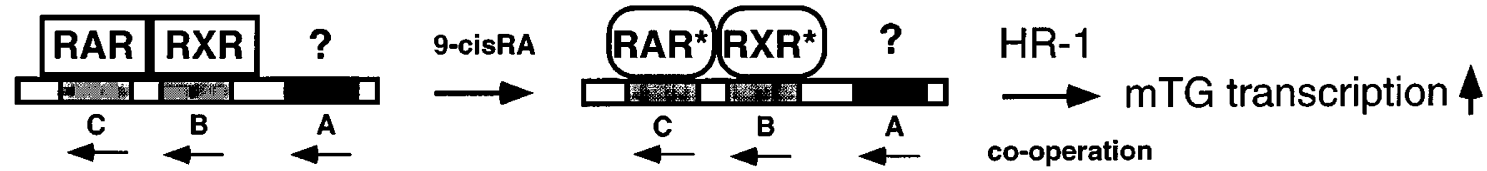

Ligand-

dependent

activation

B Activation of RXR

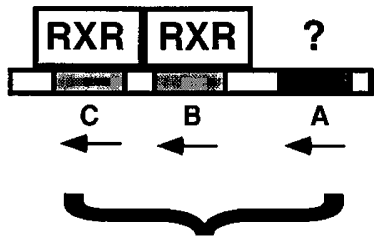

MTGRRE1

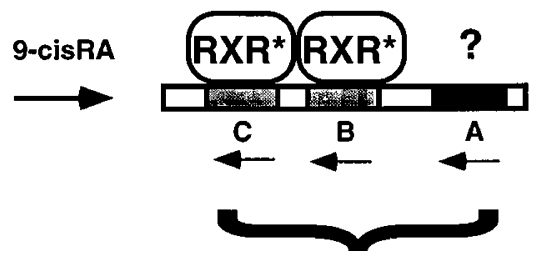

$\stackrel{\mathrm{HR}-1}{\longrightarrow} \mathrm{mTG}$ transcription $\uparrow$ co-operation

Figure 3 Retinoid regulation of tissue transglutaminase gene expression, the role of a versatile, tripartite response element (mTGRRE1) (A) RAR-RXR heterodimers are able to bind to $B$ and $C$ halfsites (DR5, direct repeat) of mTGRRE1, upon ligand activation of either the RAR side or both RAR and RXR sides transcription is initiated. (B) the DR5 element is also able to bind RXR homodimers, and confer RXR-dependent transcriptional activation upon ligand stimulation. Two additional elements contribute to retinoid regulation of the mouse tissue transglutaminase gene: the A halfsite, which forms a DR7 with $B$ and a region highly homologous in mouse and human HR-1 (homology region-1) (Nagy et al, 1996a) which is located further downstream from mTGRRE1 and necessary for full retinoid response in the context of the mouse tissue transglutaminase promoter 
1996b), ICE (caspase 1) and CPP32 (caspase 3) (Watson et al, 1997) are elevated. It is interesting to note that Bcl-2 down-regulation is RAR-mediated while induction of tissue transglutaminase is predominantly RXR-induced in this myeloid cell line (Nagy et al, 1996b). This suggests that differentiation and apoptotic cell death of these cells are intimately linked and that retinoids are able to initiate an orchestrated process which involves both positive and negative changes in gene expression of a diverse group of factors known to be associated with apoptosis.

We studied the transcriptional regulation of the apoptosis effector tissue transglutaminase in order to gain insight into the molecular mechanisms of retinoid regulated expression of this enzyme. We attempted to identify cis-acting regulatory elements which are able to direct expression upon many diverse regulatory stimuli.

Tissue transglutaminase (TGase) is an intracellular protein cross-linking enzyme that accumulates to high levels in cells undergoing apoptotic cell death (for reviews see Aeschlimann and Paulsson (1994); Greenberg et al (1991); Nagy et al (1994)). The enzyme is both induced and activated (by falling GTP and rising intracellular $\mathrm{Ca}^{2+}$ ) during apoptosis (Fesus et al, 1987, 1989). Activation of TGase in apoptotic cells, results in the extensive crosslinking of intracellular proteins during the fragmentation phase of the apoptotic program (Fesus et al, 1989). Overexpression of TGase in cells (by transfection with TGase expression vectors) results in high levels of spontaneous apoptosis (Gentile et al, 1992; Melino et al, 1994). Inhibition of TGase expression (with antisense expression vectors) results in a reciprocal decrease in apoptotic activity (Melino et al, 1994). It is thought that the formation of a densely cross-linked intracellular matrix contributes to the fragmentation of cells during apoptosis as well as preventing the leakage of intracellular proteins during the formation of apoptotic bodies (Piredda et al, 1997).

TGase is expressed in many sites of physiological apoptosis in vivo. During limb development, the enzyme accumulates in clusters of apoptotic cells in the interdigital webs as well as in the hypertrophic chondrocytes of the developing bones (Thomazy and Davies, unpublished observations). TGase also accumulates in the apoptotic hepatocytes formed during the reversal phase of druginduced hepatomegaly (Thomazy and Fesus, 1989) as well as in involuting mammary and prostate glands following removal of trophic stimuli (Fesus et al, 1991). Comparison of the results obtained with in situ hybridization probes and TGase immunochemistry suggest that TGase mRNA is expressed in cells prior to the development of morphologic evidence of apoptosis whereas the actually enzyme accumulates to high levels in cells that are well advanced in the apoptotic program (Thomazy and Davies, unpublished observations). Studies with the TGase transgene confirm that the promoter for the TGase gene is activated in cells before the appearance of an apoptotic phenotype (Nagy et al, 1997a) suggesting that the activation of the gene is an early component in the commitment of cells undergoing apoptosis.

The induction of TGase expression during in vivo apoptosis can be replicated in some, but not all forms, of apoptosis induced in vitro. The induction of apoptosis in hepatocytes (Piacentini et al, 1992), neuroblastoma cells (Melino et al, 1994), myeloid leukemia cells (Nagy et al, 1996b), trachealepithelial cells (Zhang et al, 1995) and others has been associated with the induction of tissue TGase expression. The very rapid apoptosis that develops following DNA damage, a p53-dependent form of apoptosis, is not associated with evidence of increased TGase expression ( $\mathrm{Lu}$ and Davies, unpublished observations). In summary it appears that the enzyme is induced in most forms of physiological cell death, particularly in cell death associated with morphogenesis and endocrine-regulated tissue remodelling.

The association of TGase with apoptosis has lead to considerable interest in the factors that regulate the enzyme's expression. Several years ago we and others demonstrated that retinoids were potent and specific regulators of TGase expression in vitro and in vivo (Davies et al, 1985; Moore et al, 1984). The effects of retinoids are physiologically significant since depletion of endogenous retinoids in rats, by vitamin A deficiency, results in a global decrease in the levels of TGase expressed in cells and tissues (Verma et al, 1992). We subsequently cloned the mouse and human TGases and showed that retinoids regulate transcription of the TGase gene (Chiocca et al, 1988). An unusual feature of the transglutaminase gene that it is under complex retinoid regulation. It can be activated by ligands of both RARs and RXRs in different cells and tissues (Nagy et al, 1996b). This on one hand provides a mechanism so that the enzyme's expression can be induced multiple forms of retinoid induced apoptosis on the other it raises the possibility that there may be multiple, separate cis-acting elements contributing to the gene's regulation. More recently we have isolated and characterized the promoters for both the human and mouse Tgases (Lu et al, 1995; Nagy et al, 1997a). We have identified in the mouse gene a specific tripartite retinoid response element (mTG RRE-1) (Figure 3) whose presence is required for efficient retinoiddependent activation of the promoter (Nagy et al, 1996a). The TGase RRE-1 functions as a ligand-dependent enhancer element capable of activating homologous and heterologous promoters in a position- and orientationindependent manner (Figure 3). The promoter can be activated by all three sub-types of RARs and in myeloid leukemia cells by ligands that activate endogenous RXRs (Beard et al, 1994; Nagy et al, 1996b). This profile matches that of the endogenous gene.

The isolation of the TGase promoter coupled with the observation that the gene was induced in many apoptotic cells gave us an opportunity to ask an intriguing question. Is it possible to use the TGase promoter as a targeting vector capable of selectively inducing gene expression in apoptotic cells? We coupled a $3.8 \mathrm{~kb}$ fragment of the mouse TGase promoter to a beta-galactosidase reporter gene and then used this reporter transgene to make transgenic mouse lineages (Nagy et al, 1997a). In the lineages we characterized, the transgene is selectively expressed in the embryonic limb in regions of cells undergoing apoptosis (Nagy et al, 1997a). The reporter 
gene can be detected in morphologically normal cells associated with apoptotic cells as well as in cells that are distinctly apoptotic suggesting that the transgene is actually activated early in the apoptotic program (Nagy et al, 1997a). These studies have confirmed our hypothesis that the activity of the TGase promoter is specifically linked to regions (i.e. interdigital web) of apoptotic cell death in vivo. In addition they demonstrate that all of the information required to express this gene in apoptotic cells is embedded within the proximal $3.8 \mathrm{~kb}$ of the promoter. This observation taken together with our previous finding, that retnoids are key regulators of the transcriptional activity of the TGase promoter provides the foundation for our proposal that retinoid regulation of tissue transglutaminase and apoptosis likely to be coupled in vivo.

\section{Perspectives}

During the past 10 years there has been significant advance in our understanding of how retinoids work and exert their biological effects. Several aspects of the molecular mechanisms of retinoid receptor signaling has been clarified: nuclear receptors for retinoids were cloned, natural and synthetic ligands were identified and recently several receptor interacting cofactors were discovered. Connections between retinoids and the regulation of complex processes such as differentiation and apoptotic cell death has been made both in normal development and in disease. Thousands of synthetic receptor specific retinoid analogs have been developed and tested to elucidate the function and biological activites of retinoid receptors. This has led to the development of new therapeautic approaches in the treatment of certain leukemias, squamous cell carcinomas, breast and lung cancers as well as in a variety of skin diseases. Several of these treatments utilizes the fact that retinoids are able to induce terminal differentiation and/or apoptosis of unwanted pools of malignant or rapidly proliferating cells. Although a connection between retinoids and apoptosis has been established, most of the reports on this subject concentrate on documenting the biological processes rather than dissecting the molecular components of them. More mechanistic studies are needed to clarify the molecular details of retinoid regulated apoptosis. For instance, we do not fully understand the role of endogenous retinoids and individual receptors as well as the contribution of other signaling pathways to morphogenic apoptosis. Also, identification of new target genes and dissection of the mechanisms of their retinoid regulation would shed light on the secondary regulatory components and effector elements of retinoid modulated apoptosis. Finally, it is still not clear how retinoid regulated gene expression leads to the activation of the well characterized executionary phase of apoptosis.

\section{References}

Aeschlimann D and Paulsson M (1994) Transglutaminases: protein cross-linking enzymes in tissues and body fluids. Thromb Haemost 71: 402-415

Alland L, Muhle R, Hou H, Potes J, Chin L, Schreiber-Agus N, and DePinho R (1997) Nature 387: 4955
Alles AJ and Sulik KK (1989) Retinoic-acid induced limb-reduction defects: perturbation of zones of programmed cell death as a pathogenetic mechanism. Teratology 40: 163-171

Alles AJ and Sulik KK (1990) Retinoic acid-induced spina bifida: evidence for a pathogenetic mechanism. Development 108: 73-81

Apfel C, Bauer F, Crettaz M, Forni L, Kamber M, Kaufman F, LeMotte P, Pirson W and Klaus M (1992) A retinoic acid receptor $\alpha$ antagonist selectively counteracts retinoic acid effects. Proc Natl Acad Sci USA 89: 7129-7133

Atencia R, Garcia-Sanz M, Unda F and Arechaga J (1994) Apoptosis during retinoic acid-induced differentiation of F9 embryonal carcinoma cells. J Exp Cell Res 214: 663-637

Ayer D, Lawrence $Q$ and Eisenman R (1995) Mad-Max transcriptional repression is mediated by ternary complex formation with mammalian homologs of yeast repressor Sin3. Cell 80: 767-776

Beard RL, Gil DW, Marler DK, Henry E, Colon DF, Gillett SJ, Davies PJA and Chandraratna RAS (1994) Structural basis for the differential RXR \& RAR activity of stilbene retinoid analogs. Bioorganic and Medicinal Chemistry Letters 4 : $1447-1452$

Boccia M, Xu Q, WesleyU, XuY, Korontsvit T, Loganzo F, Albino A and Scheinberg D (1997) Mudulation of p53, WAF1/p21 and BCL-2 expression during retinoic acidinduced differentiation of NB4 promyelocytic cells. Leuk Res 21: 439-447

Bourguet W, Ruff M, Chambon P, Gronemeyer H and Moras D (1995) Crystal structure of the ligand-binding domain of the human nuclear receptor RXR-alpha. Nature 375: 377-382

Chambon P (1996) A decade of molecular biology of retinoic acid receptors. FASEB Journal 10: $940-954$

Chen H, Lin R, Schiltz R, Chakravarti D, Nash A, Nagy L, Privalsky M, Nakatani Y and Evans R (1997) Nuclear receptor coactivator ACTR is a novel histone acetyltransferase and forms a multimeric activation complex with P/CAF and CBP/p300. Cell 90: 569-580

Chen J, Penco S, Ostrowski J, Balaguer P, Pons M, Starrett J, ReczekP, Chambon P and Gronemeyer H (1995) RAR-specific agonist/antagonists which dissociate transactivation and AP1 transrepression inhibit anchorage-independent cell proliferation. EMBO J 14:1187-1197.

Chen JD and Evans RM (1995) A Transcriptional Co-Repressor That Interacts With Nuclear Hormone Receptors. Nature 377: 454-457.

Chiocca EA, Davies PJ and Stein JP (1988) The molecular basis of retinoic acid action. Transcriptional regulation of tissue transglutaminase gene expression in macrophages. J Biol Chem 263: 11584-11589

Corbeil J, Rapaport E, Richman D and Looney D (1994) Antiproliferative effect of retinoid compounds on Kaposi's sarcoma cells. J Clin Invest 93: 1981-1986

Davies PJ, Murtaugh MP, Moore WT, Jr, Johnson GS and Lucas D (1985) Retinoic acid-induced expression of tissue transglutaminase in human promyelocytic leukemia (HL-60) cells. J Biol Chem 260: 5166-5174

Fanjul A, Dawson M, Hobbs P, Jong L, Cameron J, Harlev E, Graupner G, Lu X and Pfahl M (1994) A new class of retinoids with selective inhibition of AP- 1 inhibits proliferation. Nature 372: 107-111

Fanjul A, Delia D, Pierotti M, Rideout D, Qin J and Pfahl M (1996) 4-Hydroxyphenyl retinamide is a highly selective activator of retinoid receptors. $\mathrm{J}$ Biol Chem 271 $22441-22446$

Ferretti P and Geraudie J (1995) Retinoic acid-induced cell death in the wound epidermis of regenerating zebrafish fins. Dev Dyn 202: 271-283

Fesus L, Thomazy V and Falus A (1987) Induction and activation of tissue transglutaminase during programmed cell death. FEBS Lett 224: 104-108

Fesus L, Thomazy V, Autuori F, Ceru MP, Tarcsa E and Piacentini M (1989) Apoptotic hepatocytes become insoluble in detergents and chaotropic agents as a result of transglutaminase action. FEBS Lett 245: 150-154

Fesus L, Davies PJA and Piacentini M (1991) Apoptosis: molecular mechanisms in programmed cell death. European Journal of Cell Biology 56: 170-177

Gentile V, Thomazy V, Piacentini M, Fesus L and Davies PJ (1992) Expression of tissue transglutaminase in Balb-C 3T3 fibroblasts: effects on cellularmorphology and adhesion. J Cell Biol II9: 463-474

Greenberg CS, Birckbichler PJ and Rice R H (1991) Transglutaminases: multifunctional cross-linking enzymes that stabilize tissues. FASEB J 5 $3071-307$

Gudas L, Sporn M and Roberts A (1994) Cellular biology and biochemistry of the retinoids. In The Retinoids. Biology, Chemistry and Medicine, M Sporn, A Roberts and D Goodman, eds (New York: Raven Press) 
Hassig C, Fleischer T, Billin A, Schreiber S and Ayer D (1997) Histone deacetylase activity is required for full transcriptional repression by mSin3A. Cell 89: $341-$ 347

Heinzel T, Lavinsky R, Mullen T-M, Soderstrom M, Laherty C, Torchia J, Yang W-M, Brard G, Ngo S, Davie J, Glass C and Rosenfeld M (1997) N-CoR, mSin3 and histone deacetylase-containing complexes in nuclear receptor and Mad repression. Nature 387: 43-48

Horlein AJ, Naar AM, Heinzel T, Torchia J, Gloss B, Kurokawa R, Ryan A, Kamel Y, Soderstrom M, Glass CK and Rosenfeld MG (1995) Ligand-Independent Repression By the Thyroid Hormone Receptor Mediated By a Nuclear Receptor Co-Repressor. Nature 377: 377-404

Jetten A, Kim J, Sacks P, Rearick J, Lotan D, Hong W and Lotan R (1990) Inhibition of growth and squamous-cell differentiation markers in cultured human head and neck squamous carcinoma cells by beta-all-trans retinoic acid. Int J Cancer 45 : 195-202

Jiang $\mathrm{H}$ and Kochhar DM (1992) Induction of tissue transglutaminase and apoptosis by retinoic acid in the limb bud. Teratology 46: $333-40$

Johnson A, Klein E, Gillett S, Wang L, Song T, Pino M and Chandraratna R (1995) Synthesis and characterization of a highly potent and effective antagonist of retinoic acid receptors. J Med Chem 38: 4764-4767

Kalemkerian G, Slusher R, Ramalingam S, Gadgeel S and Mabry M (1995) Growth inhibition and induction of apoptosis by fenretinide in small -cell lung cancers cell lines. J NCl 87: 1676-1680

Kamei Y, Xu L, Heinzel T, Torchia J, Kurokawa R, Gloss B, Lin S, Heyman R, Rose D, Glass C and Rosenfeld M (1996) A CBP integrator complex mediates transcriptional activation and AP-1 inhibition by nuclear receptors. Cell 85 : $403-414$

Kastner P, Mark M and Chambon P (1995) Nonsteroid nuclear receptors - What are genetic studies telling us about their role in real lif. Cell 83: 859-869

Kochar D (1967) Teratogenic activity of retinoic acid. Acta Pathologica and Microbiologica Scandinavica 70: 398-404

Krupitza G, Hulla W, Harant H, Dittrich E, Kallay E, Huber H, Grunt T and Dittrich C (1995) Retinoic acid induced death of ovarian carcinoma cells correlates with cmyc stimulation. Int $\mathrm{J}$ of Cancer 61: 649-657

Kurokawa R, DiRenzo J, Boehm M, Sugarman J, Gloss B, Rosenfeld MG, Heyman RA and Glass CK (1994) Regulation of retinoid signalling by receptor polarity and allosteric control of ligand binding. Nature 371: 528-531

Kurokawa R, Soderstrom M, Horlein A, Halachmi S, Brown M, Rosenfeld MG and Glass CK (1995) Polarity-Specific Activities Of Retinoic Acid Receptors Determined By a Co-Repressor. Nature 377: 451-454

Lee KKH, Li FCH, Yung WT, Kung JLS, Ng JL and Cheah KSE (1994) Influence Of Digits, Ectoderm, and Retinoic Acid On Chondrogenesis By Mouse Interdigital Mesoderm In Culture. Developmental Dynamics 201: 297-309

Li E, Sucov H, Lee K, Evans R and Jaenisch R (1993) Normal development and growth of mice carrying a targeted disruption of the alpha 1 retinoic acid receptor gene. Proc Natl Acad Sci USA 90: 1590-1594

Lin M, lawarone A and Freedman L (1996) Retinoid induction of U937 cell differentiation: transcriptional activation of the human p21WAFI/CIP1 gene by retinoic acid receptor. J Biol Chem 271: 31723-31728

Lohnes D, Kastner P, Dierich A, Mark M, LeMeur M and Chambon P (1993) Function of retinoic acid receptor gamma in the mouse. Cell 73: 643-658

Lu S, Saydak M, Gentile V, Stein JP and Davies PJA (1995) Isolation and characterization of the human tissue transglutaminase gene promoter. J. Biol. Chem., 271: 9748-9756

Lufkin T, Lohnes D, Mark M, Dierich A, Gorry M, Gaub P, LeMeur M and Chambon P (1993) High post natal lethality and testis degeneration in retinoic acid receptor alpha mutant mice. Proc Natl Acad Sci 90: 7225-7229

Luo J, Pasceri P, Conlon R, Rossant J and Giguere V (1995) Mice lacking all iso-forms of retinoic receptor beta develop normally and are susceptible to the teratogenic effects of retinoic acid. Mech Dev 53: 61-71

Mangelsdorf DJ, Umesono K and Evans RM (1994) The retinoid receptors. In The retinoids, MB Sporn, AB Roberts and DS Goodman, eds (New York: Raven Press), pp. 319-350

Martin SJ, Bradley JG and Cotter TG (1990) HL-60 cells induced to differentiate towards neutrophils subsequently die via apoptosis. Clinical Experimental Immunology 79: 448-453

Melino G, Annicchiarico-Petruzzelli M, Piredda L, Candi E, Gentile V, Davies PJ and Piacentini M (1994) Tissue transglutaminase and apoptosis: sense and antisense transfection studies with human neuroblastoma cells. Mol Cell Bio 14: $6584-6596$
Melino G, Draoui M, BellincampiL, BernassolaF, Bernardini S, Piacentini M, Reicher $U$ and Cohen P (1997) Retinoic acid receptors $\alpha$ and $\gamma$ mediate the induction of tissue transglutaminase activity and apoptosis in human neuroblastoma cells. Exp Cell Res 235: 55-61

Mendelsohn C, Ruberte E, LeMeur M, Morriss-Kay G and Chambon P (1991) Developmental analysis of the retinoic acid -inducible RAR-beta 2 promoter in transgenic animals. Development 113: 723-34

Moore WT, Jr, Murtaugh MP and Davies PJ (1984) Retinoic acid -induced expression of tissue transglutaminase in mouse peritoneal macrophages. J Biol Chem 259 $12794-802$

Nagpal S, Athanikar J and Chandraratna R (1995) Separation of transactivation and AP1 antagonism functions of retinoic acid receptor alpha. JBiol Chem 270: 923 927

Nagy L, Thomazy VA and Davies PJA (1994) Tissue transglutaminase: an effector in physiologic cell death. Cancer Bulletin 46: 136-140

Nagy L, Thomazy VA, Shipley GL, Fesus L, Lamph W, Heyman RA, Chandraratna RAC and Davies PJA (1995) Activation of Retinoid X Receptors (RXR) induces apoptosis in HL-60 cell lines. Mol Cell Biol 15: 3540-3551

Nagy L, Saydak M, Shipley N, Lu S, Basilion JP, Yan ZH, Syka P, Chandraratna R, Heyman R and Davies PJA (1996a) Identification and characterization of a versatile retinoid response element (RARE/RXRE) in the promoter of the mouse tissue transglutaminase gene. J Biol Chem 271: 4355-4365

Nagy L, Thomazy VA, Heyman RA, Chandraratna RAS and Davies PJA (1996b) Retinoid-regulated expression of $\mathrm{BCL}-2$ and tissue transglutaminase during differentiation and apoptosis of human myeloid leukemia (HL-60) cells Leukemia Research 19: 499-505

Nagy L, Thomazy V, Saydak M, Stein J and Davies P (1997a) The promoter of the mouse tissue transglutaminase gene directs tissue-specific, retinoid-regulated and apoptosis-linked expression. Cell Death Differ 4: 534547

Nagy L, Kao H-Y, Chakravarti D, Lin R, Hassig C, Schreiber S, Ayer D and Evans R (1997b) Nuclear receptor repression mediated by a complex containing SMRT, mSin3A, and histone deacetylase. Cell 89: 373-380

Nakamura N, Shidoji Y, Yamada Y, Hatakeyama H, Moriwaki H and Muto Y (1995) Induction of apoptosis by a cyclic retinoid in the human hepatoma derived cell line Huh-7. Biochem. Biophys. Res. Com. 207: 328-388

Ogryzko V, Schiltz R, Russanova V, Howard B and Nakatani Y (1996) The transcriptional coactivators p300 and CBP are histone acetyltransferases. Cell 87: 953-959

Oridate N, Lotan D, Mitchell M, Hong W and Lotan R (1995) Induction of apoptosis by retinoids in human cervical carcinoma cell lines. International $\mathrm{J}$ of Oncology 7 : $433-441$

Perlmann T and Evans R(1997) Nuclear receptors in Sicily: all in the famiglia. Cell 90 $391-397$

Piacentini M, Ceru MP, Dini L, Di Rao M, Piredda L, Thomazy V, Davies PJA and Fesus $L$ (1992) In vivo and in vitro induction of tissue transglutaminase in rat hepatocytes by retinoic acid. Biochimica et Biophysica Acta, 171-179

Piredda L, Amendolo A, Colizzi V, Davies P, Farrace MG, Fraziano M, Gentile V, Uray I, Piacentini M and Fesus L (1997) Lack of tissue transglutaminase protein crosslinking leads to leakage of macromolecules from dying cells: relationship to development of autoimmunity in MRL Ipr/lpr mice. Cell Death Differ 4: 463-472

Renaud J, Rochel N, Ruff M, Vivat V, Chambon P, Gronemeyer Hand Moras D (1995) Crystal structure of the RAR gamma ligand binding domain bound to all trans retinoic acid. Nature 378: 681-689

Riboni L, Prinetti A, Bassi R, Caminiti A and Tettamanti G (1995) A mediator role of ceramide in the regulation of neuroblastoma Neuro2a cell differentiation. J Biol Chem 270: 26868-26875

Robertson KA, Emami B and Collins SJ (1992a) Retinoic acid-resistant HL-60R cells harbor a point mutation in the retinoic acid receptor ligand-binding domain that confers dominant negative activity. Blood 80: 1885-1889

Robertson KA, Emami B, Mueller L and Collins SJ (1992b) Multiple members of the retinoic acid receptor family are capable of mediating the granulocytic differentiation of HL-60 cells. Mol. Cell. Biol. 12: $3743-3749$

Roy D, Taneja R and Chambon P (1995) Synergistic activation of retinoic acid responsive genes and induction of embryonal carcinoma cell differentiation by an RARalpha RARbeta or RARgamma-selective ligand in combination with retinoid $X$ receptor-selective ligand. Mol Cell Biol 15: 6481-6487

Schule R, Rangarajan P, Yang N, Kliewer S, Ransone L, Bolado J, Verma I and Evans $R$ (1991) Retinoic acid is a negative regulator of AP-1-responsive genes. Proc Natl Acad Sci USA 88: 6092-6096 
Seewald V, BS Parker M, Collins S and Swisshelm K (1995) Expression of retinoic acid beta mediates retinoic acid-induced groth arrest and apoptosis in breast cancer cells. Cell Growth and Diff 6: 1077-1088

Sheikh M, Shao Z, LiX, Ordonez J, Conley B, Wu S, Dawson M, Han Q, Chao W, Quick T, Niles R and Fontana J (1995) N-(4-Hydroxyphenyl)Retinamide(4-Hpr-)Mediates biological actions involve retinoid receptor-independent pathways in human breast carcinoma. Carcinogenesis 16: 2477-2486

Soprano D, Gyda M, Jiang H, Harnish D, Ugen K, Satre M, Chen L, Soprano K and KocharD (1994) A sustained elevation in retinoic acid receptor-beta2 mRNA and protein occures during retinoic-acid induced fetal dysmorphogenesis. Mech of Dev 45: 243-253

Sulik K, Sulik KK, Cook C and Webster W (1988) Teratogenes and craniofcial malformations Develpoment 102: suppl, 213-231

Sulik KK and Dehart DB (1988) Retinoic-acid-induced limb malformations resulting from apical ectodermal ridge cell death. Teratology 37: 527-537

Szondy Z, Reichert U, Branardon J, Michel S, Toth R, Ancian P, Ajzner E and Fesus L (1997) Induction of apoptosis by retinoids and RARg selective compounds in mouse thymocytes through a novel apoptosis pathway. Mol Pharmacol 51:972 982

Taneja R, Bouillet P, Boylan J, Baub M, Roy L, Gudas L and Chambon P (1995) Reexpression of retinoic acid receptor gamma or overexpression of RAR-alpha or RAR-beta in RAR-gamma null F9 cells reveals a partial functional redundancy between the three RAR types. Proc Natl Acad Sci USA 92: 7854-7858
Thomazy V and Fesus L (1989) Differential expression of tissue transglutaminase in human cells. An immunohistochemical study. Cell Tissue Res 255: 215-224

Verma AK, Shoemaker A, Simsiman R, Denning M and Zachman RD (1992) Expression of retinoic acid nuclear receptors and tissue transglutaminase is altered in various tissues of rats fed a vitamin A-deficient diet. J Nutr 122: 2144 2152

Watson R, Rothstein O, Parodo J, Bitar R, Hackam D and Marshall J (1997) Granulocytic differentiation of HL-60 cells results in spontaneous apoptosis mediated by increased caspase expression. FEBS Lett. 412: 603-609

Wilson J, Roth C and Warkany J (1953) An analysis of the syndrome of malformations induced by maternal vitamin A deficiency. Effects of restoration of vitamin A at various times during gestation. Am J of Anatomy 92: 189-217

Wolbach S and Howe P (1925) Tissue changed following deprivation of fat-soluble vitamin A. Journal of Exp Med 42: 753-780

Yang Y, Vaccio M and Ashwell J (1993) 9-cis retinoic acid inhibits activation-driven Tcell apoptosis: implications for retinoid $\mathrm{x}$ receptor involvement in thymocyte development. Proc Natl Acad Sci USA 90: 6170-6174

Zakeri ZF and Ahuja HS (1994) Apoptotic Cell Death In the Limb and Its Relationship to Pattern Formation. Biochemistry \& Cell Biology 72: 603-613

Zhang LX, Mills KJ, Dawson MI, Collins S J and Jetten AM (1995) Evidence for the involvement of retinoic acid receptor RAR alpha-dependent signaling pathway in the induction of tissue transglutaminase and apoptosis by retinoids. J Biol Chem 270: $6022-6029$ 